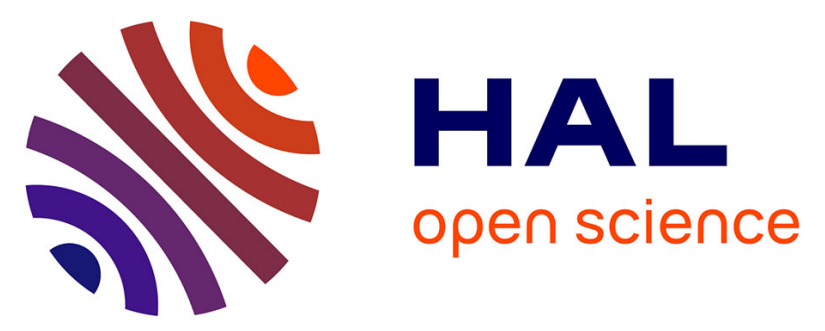

\title{
Taking into Account GTEM Field Nonuniformities in Radiated Immunity Simulations Through a Simple Measurement
}

\author{
Sjoerd Op 'T Land, Richard Perdriau, Mohamed Ramdani
}

\section{To cite this version:}

Sjoerd Op 'T Land, Richard Perdriau, Mohamed Ramdani. Taking into Account GTEM Field Nonuniformities in Radiated Immunity Simulations Through a Simple Measurement. IEEE Transactions on Electromagnetic Compatibility, 2017, 59 (6), pp.2042-2045. 10.1109/TEMC.2017.2691800 . hal01622381

HAL Id: hal-01622381

https://hal-univ-rennes1.archives-ouvertes.fr/hal-01622381

Submitted on 1 Feb 2018

HAL is a multi-disciplinary open access archive for the deposit and dissemination of scientific research documents, whether they are published or not. The documents may come from teaching and research institutions in France or abroad, or from public or private research centers.
L'archive ouverte pluridisciplinaire HAL, est destinée au dépôt et à la diffusion de documents scientifiques de niveau recherche, publiés ou non, émanant des établissements d'enseignement et de recherche français ou étrangers, des laboratoires publics ou privés. 


\title{
Taking into Account GTEM Field Nonuniformities in Radiated Immunity Simulations through a Simple Measurement
}

\author{
Sjoerd Op 't Land ${ }^{* \dagger}$, Member, IEEE, Richard Perdriau*†, Senior Member, IEEE, Mohamed Ramdani*†, Senior \\ Member, IEEE \\ ${ }^{*}$ RF-EMC Research Group, ESEO-TECH, \\ Angers, France \\ Email: \{sjoerd.optland, mohamed.ramdani, richard.perdriau $\} @$ eseo.fr \\ $\dagger$ ADH, IETR \\ Rennes, France
}

\begin{abstract}
When performing immunity measurements through the $10 \times 10-\mathrm{cm}$ aperture of a GTEM (Gigahertz Transverse ElectroMagnetic) cell, its field uniformity is generally unknown. Yet, this (non)uniformity causes an error on test results. Measuring the field uniformity by taking a grid of samples would be timeconsuming. Even with such a measurement, it would be hard to predict the magnitude of this error in immunity measurement results. Therefore, another approach to the problem was taken: several imperfections of the GTEM cell were characterized simultaneously by measuring its $S_{11}$-parameter. Then, the immunity of a DUT (Device Under Test) was simulated with the nominal GTEM field plus the first reflection. Entering this field model into the simulation corrected the major error in the $0.1-2.0 \mathrm{GHz}$ range in some of our test cases, with no degradation in any case. Thanks to the speed of this method, it may be broadly applied, even only to quantify the effect of some of the GTEM's imperfections on a given immunity measurement.
\end{abstract}

Index Terms-GTEM cell, field uniformity, modeling, simulation, ROME (Return On Modeling Effort), field-to-trace coupling, measurement uncertainty

\section{INTRODUCTION}

In previous research, Op 't Land et al. proposed a model for the coupling of a plane wave to a PCB (Printed Circuit Board) trace [1]. To challenge the model, they confronted it to measurements where the GTEM cell was used to emulate the plane wave. Often, a good fit was obtained, but sometimes, a wildly fluctuating error up to some $\mathrm{GHz}$ appeared, as shown in the example of Figure 1.

The authors know that the GTEM cell is an imperfect plane wave emulator. However, the field-to-trace-coupling model was rather new. Therefore, additional proof was needed that the problem lie in the GTEM cell, and not in the model. A full-wave simulation was run, which closely resembled the simulation of the model [2]. Yet, still more proof was needed.

The first suspect was the field nonuniformity of the GTEM cell. Unfortunately, no specifications were available on the field nonuniformity under the $10 \times 10-\mathrm{cm}$ aperture. Even if the amplitude of the field uniformity would have been known (e.g. $\pm 6 \mathrm{~dB}$ for the "working volume" [3]), what would its final effect on the results have been? If the DUT had been small with respect to the spatial field variations, the field could have been considered locally uniform. Consequently, the final effect should have been proportional to the field uniformity (e.g. $\pm 6 \mathrm{~dB})$. However, there is no specification on the spatial size of the field variations, so there is no proof that the field can be considered locally uniform. Therefore, no conclusions can be drawn on the final effect of the field nonuniformity.

For these reasons, the authors set out to better understand the field uniformity of the GTEM cell.

\section{A. State of the Art}

In the eighties, the GTEM cell was developed as a compact and broadband (DC-18 GHz) alternative to the OATS (OpenArea Test Site) [4].

The GTEM cell, presented in Figure 2, is a rectangular, paraxial and tapered $50-\Omega$ waveguide. Its center conductor is called the septum. At the small side, the GTEM cell has a coaxial input. At the large side, there is a hybrid termination: a discrete $50-\Omega$ resistor network, combined with pyramidal foam absorbers.

In theory, incoming electromagnetic power is converted into a single mode: TEM. The wave then propagates from the small to the large side, to be entirely absorbed by the termination. Consequently, the amplitude of the septum voltage $V_{\text {septum }}$ is uniform all over the septum. Of course, its phase diminishes in the propagation direction $+z$. The height between the $10 \times$ $10-\mathrm{cm}$ aperture and the septum $h_{\text {septum }}$ is almost uniform all

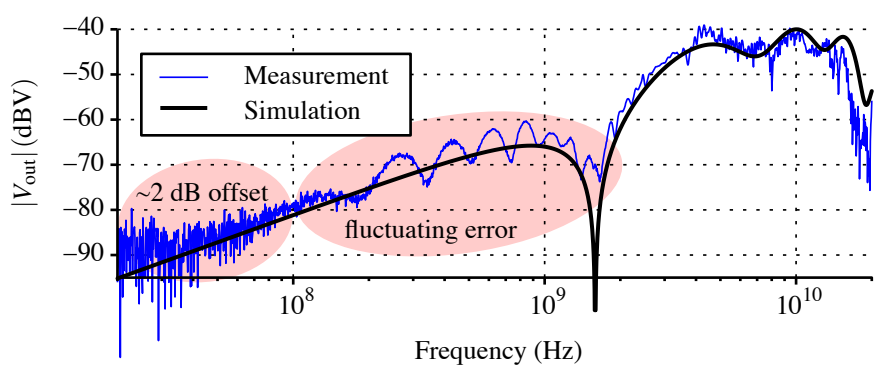

Figure 1. Example error on trace terminal voltage $V_{\text {out }}$, between measurement in a GTEM cell and a simulated new model [2, Fig. 10, South]. 
over the aperture: $42 \pm 4 \mathrm{~mm}$ due to the taper. Therefore, the electric field just under the aperture is also considered uniform all over the aperture: $V_{\text {septum }}(z) / h_{\text {septum }}$. That is: uniform field amplitude, with a phase that diminishes in the $+z$-direction.

In practice, it turns out the field is less simple, because of multiple modes and an imperfect termination. Throughout the years, the termination has been improved based on numerical [5] as well as analytical [6] approaches. Not all proposed improvements became commercially available, such as Krause and Mönich's termination, composed of ferrite absorbers and an RC network [7]. At the turn of the century, Harrington stated that the termination behaves almost perfectly, and that the remaining reflections are due to the tapered form of the GTEM cell. As the taper is fundamental to the GTEM cell, he proposed to stop trying to improve the GTEM cell, but rather take into account its actual [imperfect] behaviour [8].

\section{B. Contribution}

This letter will take Harrington's advice to heart: the imperfect behavior of the GTEM cell will be measured and taken into account in simulation.

The novelty of this letter is the simplicity of the measurement to model the GTEM imperfections ( $S_{11}$ parameter only) and the demonstrated effectiveness.

\section{Letter Structure}

The GTEM model and the method to simulate this model will be presented in Section II. This modeling and simulation method will then be applied to several case studies, the results of which will be presented in Section III. These particular results will lead to a general conclusion in Section IV. The limitations of these conclusions will be transformed into perspectives in Section V.

\section{Model AND Simulation}

Recall the initial problem: knowing the nonuniform GTEM field under the $10 \times 10-\mathrm{cm}$ aperture, in order to predict the effect of the nonuniformity on immunity measurement results.

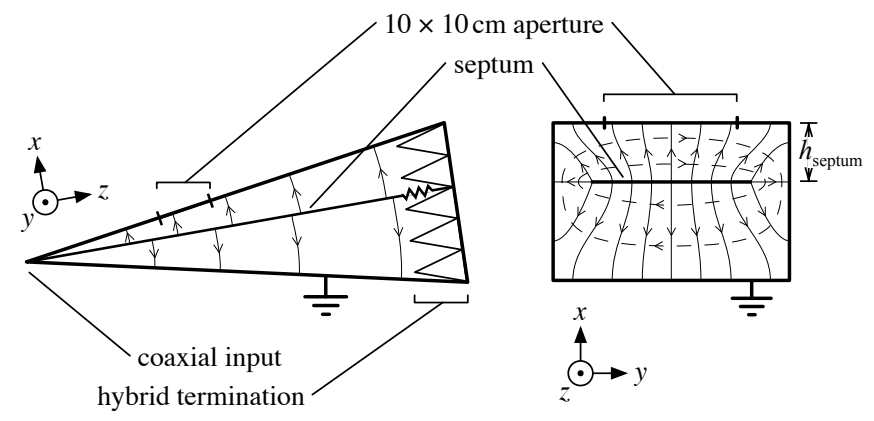

(a)

(b)

Figure 2. The GTEM cell with its electric field (solid arrows), magnetic field (dashed arrows) and coordinate system used in this letter, not to scale. (a) Lateral cross-section. (b) Frontal cross-section.

\section{A. Model's Rationale}

The GTEM field nonuniformity is caused by the GTEM cell's taper and its termination, amongst others (cf. Section I-A). Both contributors could be described as transmission line reflections, if we suppose TEM to be the dominant mode. Because the termination and the longer part of the taper are beyond the aperture, they may be lumped together in one mismatched load $Z_{\text {term }}$ just beyond the aperture. Notice that this load really is a model: it describes several phenomena in one, simplified reflection coefficient $\Gamma_{\text {term }}$. Figure 3 presents the resulting model of the GTEM cell.

As the transmission line between the GTEM cell's input and the aperture is modeled as being perfect, $\Gamma_{\text {term }}$ can be observed from the input. That is, the $S_{11}$ parameter can be measured with a VNA (Vector Network Analyzer) and the reference plane shifted to the aperture, by roughly measuring the distance between the input and the aperture.

\section{B. Simulation Method}

To predict the effect of the nonuniform field on the immunity measurement results, a simulation of the immunity measurement is needed. This simulation has the field as input and some observable immunity result as output.

If the simulation is a linear function, the effect of the field nonuniformity can be predicted by superposition. The simulation is run once for the incident plane wave $\left(E_{\text {inc }}\right.$ going forward $)$ and once for the reflected plane wave $\left(E_{\text {refl }}=\Gamma_{\text {term }} E_{\text {inc }}\right.$ going backward). By summing both simulation results, the observable result is predicted, as shown in Figure 4.

If no $S_{11}$ measurement is available, still the (maximum) SWR (Standing Wave Ratio) may be known from the GTEM cell specifications. Consequently, $\left|\Gamma_{\text {term }}\right|$ and $\left|\vec{E}_{\text {refl }}\right|$ can be calculated, albeit without phase information. If the second immunity simulation is run with this reflected field strength, the magnitude of the observable is still valid. The phase uncertainty means that the observables from both immunity simulations may add up in phase or out of phase. This way, the maximum and minimum values of an uncertainty interval may be calculated, respectively.

Note that the method can also be applied to non linear simulations. For example, the full-wave simulation of a non linear system can be excited by two plane wave sources

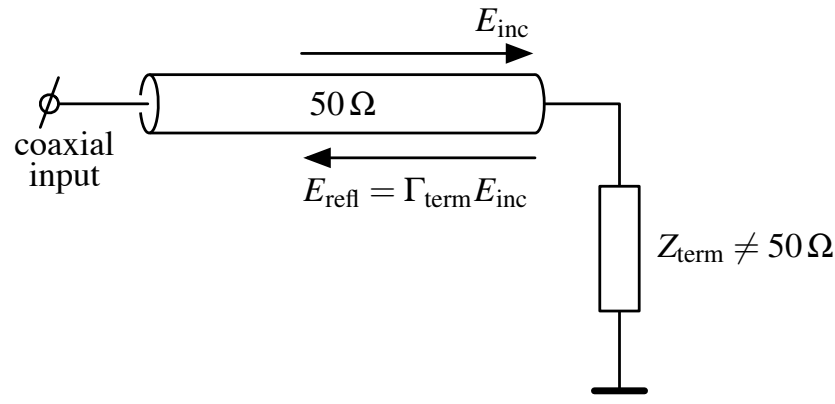

Figure 3. The proposed model of the GTEM cell: a perfect transmission line, followed by an imperfect termination. Consequently, there is precisely one reflection to consider: $\Gamma_{\text {term }} E_{\text {inc }}$. 
simultaneously $\left(E_{\text {inc }}\right.$ and $\left.E_{\text {refl }}\right)$. If the phase of $\Gamma_{\text {term }}$ were unknown, the non linear simulation could be run with a Monte Carlo sweep of $\angle \Gamma_{\text {term }}$.

\section{RESULTS}

The GTEM modeling and simulation presented above were applied to several test cases that could be measured in a GTEM cell and simulated linearly.

\section{A. Test Cases}

The immunity simulation predicted the terminal voltage at one end of a PCB trace (the observable), illuminated by an incident plane wave. For the simulation, a modified Taylor cell was used, which predicts the coupling of a vertically polarized, grazing incident plane wave to a multi-segment trace of constant characteristic impedance, with arbitrary terminal loads [2].

The immunity measurements were performed on $50-\Omega$ traces on a $10 \times 10-\mathrm{cm}$ PCB, illuminated by the GTEM cell. The terminal voltages of these traces could be observed through SMA connectors outside the GTEM cell. An example trace is shown in Figure 5.

To challenge the model, several different configurations were tried: the number of segments was chosen to be one or three, the terminal impedances were shorted, characteristic or $270 \Omega$ and the PCB was rotated in all four cardinal directions. Where possible, measurements were taken at either side of the trace, totalling 20 different immunity measurements.

\section{B. Immunity and GTEM Measurements}

In each of these immunity measurements, the voltage transfer from the septum to trace terminal was measured with a VNA as (an interpretation of the) $S_{21}$ parameter. More details about the measurement set-up and method can be found in [2].

Because the VNA was already calibrated and connected to the GTEM cell, its $S_{11}$ parameter could be measured easily. This $S_{11}$ measurement sufficed as $\Gamma_{\text {term }}$ model without phase.

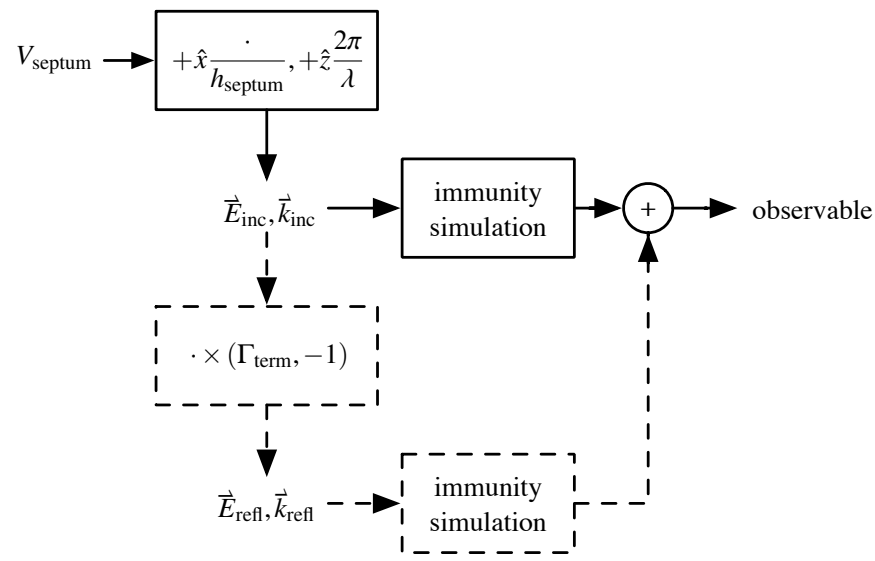

Figure 4. Taking into account the GTEM model by two linear simulations: a first time for the incident wave (solid) and a second time for the reflected wave (dashed).
To have a $\Gamma_{\text {term }}$ model with meaningful phase, the reference plane needed to be shifted from the GTEM cell input to the aperture position. From the outside of the GTEM cell, the length of the coaxial input adapter was measured to be about $8 \mathrm{~cm}$, followed by a taper of about $42 \mathrm{~cm}$ until the aperture. Without any further information about the dielectric in the coaxial input, a velocity factor of 0.7 was assumed (corresponding to a PTFE dielectric). As the taper is surrounded by air, a velocity factor of 1.0 was used. The total delay from input to aperture was thus estimated to be $1.8 \mathrm{~ns}$. This delay was subtracted from the $S_{11}$ measurement, to obtain a $\Gamma_{\text {term }}$ model with meaningful phase.

\section{Improved Simulation}

Both $\Gamma_{\text {term }}$ models (with and without phase) were applied in the method outlined in Section II-B for all 20 cases.

In some cases, significant improvement was obtained, like in the case presented in Figure 1: a three-segment trace with matched loads. By taking into account $\Gamma_{\text {term }}$ (with and without phase), the simulation results of Figure 6 a were obtained. The $2 \mathrm{~dB}$ offset at lower frequencies and noisy error above $1 \mathrm{GHz}$ remain. However, a striking resemblance of the fluctuations between $100 \mathrm{MHz}$ and $1 \mathrm{GHz}$ can be observed. Apparently, the GTEM imperfections captured in $\Gamma_{\text {term }}$ explain those fluctuations.

In other cases, only little improvement is gained. Consider for example a three-segment trace with a short circuit at one end, and $270 \Omega$ at the other end. The simulation result for this case is shown in Figure 6b. Indeed, the fluctuations of a few $\mathrm{dB}$ between 100 and $600 \mathrm{MHz}$ can be recognized, which are correctly predicted by the simulation. The original simulation that does not take into account $\Gamma_{\text {term }}$ is not shown for readability. It can be imagined halfway the gray uncertainty band. Indeed, taking into account $\Gamma_{\text {term }}$ only makes little difference. Nonetheless, this also is a meaningful result: in this

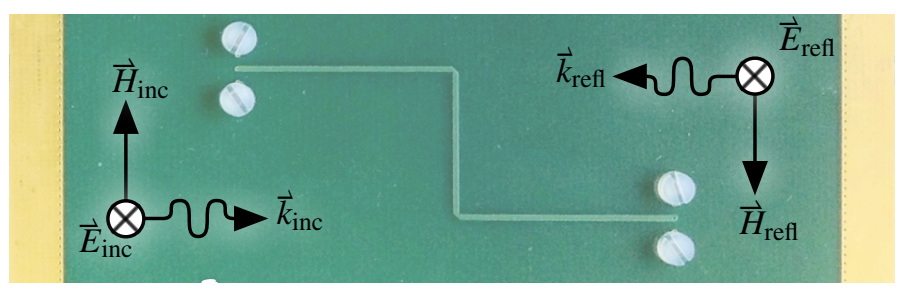

(a)

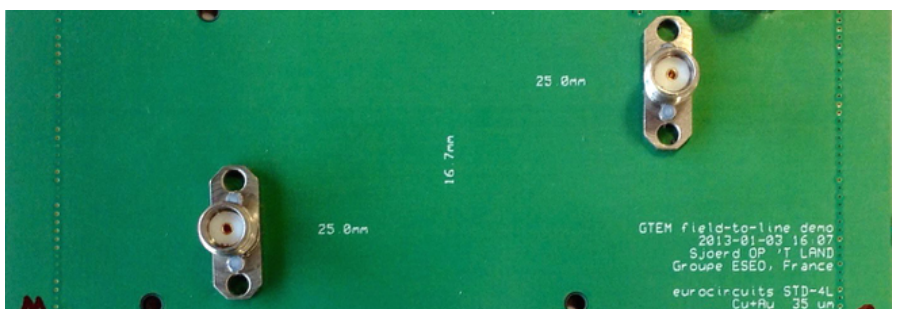

(b)

Figure 5. Detail of the PCB to be clamped upon the $10 \times 10-\mathrm{cm}$ aperture of the GTEM cell. (a) Side facing the GTEM field with a three-segment trace. (b) Side facing outward, providing SMA connectors at either end of the trace. 


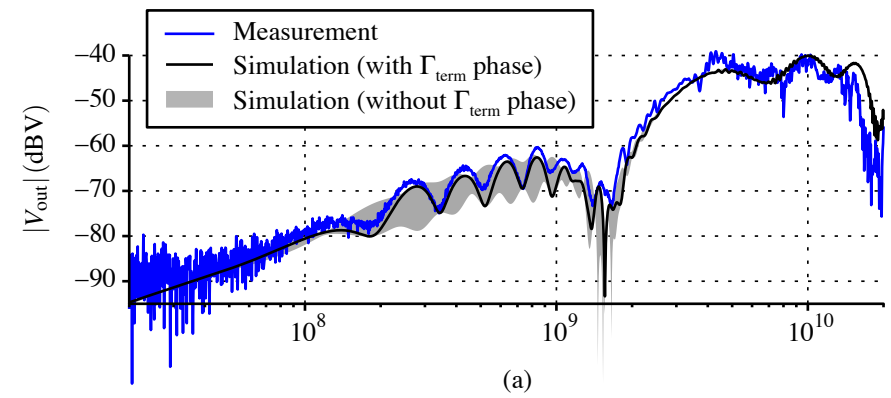

(a)

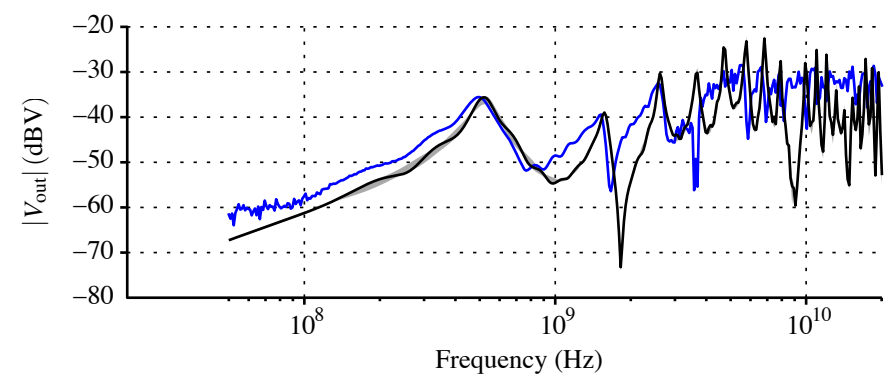

(b)

Figure 6. Results of taking into account the $\Gamma_{\text {term }}$ model of the GTEM imperfections in simulating the coupling of $1 \mathrm{~V}$ at the septum to one terminal of a three-segment PCB trace. (a) With matched loads at either end of the trace. (b) With a short circuit at one terminal, and $270 \Omega$ at the other terminal.

particular case, the influence of some GTEM cell imperfections are not the major contributor to the discrepancy between immunity measurement and simulation. Put differently: in this case, the measurement uncertainty due to these GTEM cell imperfections can be quantified to amount $\pm 1 \mathrm{~dB}$ (the height of the gray uncertainty band). Fortunately, it can be noted that the error between the measurement and the simulation has not increased by including the GTEM model.

\section{CONCLUSION}

In this paper, a method for including some GTEM imperfections into radiated immunity simulations was presented. This method only requires the measurement of the GTEM cell's $S_{11}$ parameter. Either this uncorrected measurement or the manufacturer's specification of the SWR is enough to simulate the uncertainty on the immunity measurement result. If the uncertainty is not acceptable in a given application, the measurement must be corrected by phase shifting the reference plane from the GTEM input to the DUT. This $S_{11}$ parameter with meaningful phase allows to calculate the (deterministic) error on the immunity measurement result.

In our cases, this deterministic correction never increased the simulation-measurement discrepancy. It even decreased the discrepancy in several cases, removing the major error in the 0.1 to $1 \mathrm{GHz}$ range (cf. Figure 1 vs. Figure 6a).

The $S_{11}$ measurement hardly took extra time, because in our cases, the VNA was already connected to the GTEM cell. To improve the radiated immunity simulation with this measurement would only take in the order of hours, now that the method is established. In some of our cases, significant improvement of the simulation was obtained. Therefore, depending on the economic interest of an improved radiated immunity simulation, the proposed method may have a good ROME (Return On Modeling Effort).

\section{Perspectives}

However, the uncertainty on the ROME may explain the difficulty EMC engineers have to convince their management of the importance of modeling. EMC scientists also have trouble obtaining grants, because they cannot quantify the economic impact of their research. Therefore, future research should first find several real-life EMC problems that have a known cost. Then, the potential cost saving thanks to modeling can be estimated. Finally, the net gain in modeling can be calculated, i.e. the ROME.

If economic interest is a trustworthy image of scientific value, the ROME can then be used to steer further research. If the ROME of the method presented in this paper turns out to be negative, trying to remove the few remaining dB's of error is wasted effort. This research topic should then be abandoned. If it is positive, however, further research is needed. The reallife EMC problems leading to this conclusion will then focus the remaining research. For example, the low frequency $2 \mathrm{~dB}$ offset may turn out futile, but the error at $1.5 \mathrm{GHz}$ may prove crucial for the safety of aeronautical navigation systems.

\section{ACKNOWLEDGEMENTS}

This research project was co-financed by the French national project SEISME (simulation of emissions and immunity of electronic systems).

\section{REFERENCES}

[1] S. T. Op 't Land, M. Ramdani, R. Perdriau, M. Leone, and M. Drissi, "Simple, Taylor-based worst-case model for field-to-line coupling", JPIER, vol. 140, pp. 297-311, Jun. 2013. DOI: 10.2528/PIER13041207.

[2] S. T. Op 't Land, M. Ramdani, R. Perdriau, M. Drissi, and F. Leferink, "Field-to-long-segmented-trace coupling with arbitrary loads and a transparent upper bound using a single modified Taylor cell", IEEE Transactions on Electromagnetic Compatibility, vol. 58, no. 5, pp. 1517-1525, Jul. 2016. DOI: 10.1109/TEMC.2016.2566449.

[3] IEC 61000-4-20: Electromagnetic compatibility (EMC) - part 4-20: Testing and measurement techniques - emission and immunity testing in transverse electromagnetic (TEM) waveguides, 2010.

[4] D. Königstein and D. Hansen, "A new family of TEM-cells with enlarged bandwidth and optimized working volume", in Proc. 7th Zurich Symposium and Technical Exhibition on EMC, Zurich, Switzerland., 1987, pp. 172-132.

[5] D. Hansen, D. Ristau, W. Radasky, and K. Smith, "Sources of problems in the GTEM field structure and recommended solutions", in Electromagnetic Compatibility, 1996. Symposium Record. IEEE 1996 International Symposium on, 1996, pp. 48-51. DOI: 10.1109/ISEMC. 1996.561199.

[6] R. De Leo, L. Pierantoni, T. Rozzi, and L. Zappelli, "Accurate analysis of the GTEM cell wide-band termination", IEEE Transactions on Electromagnetic Compatibility, vol. 38, no. 2, pp. 188-197, May 1996. DOI: $10.1109 / 15.494623$.

[7] J. Krause and G. Mönich, "FRCTEM cell. approaches to improve VSWR and to suppress longitudinal components in conical TEM cells", in Electromagnetic Compatibility, 1997. IEEE 1997 International Symposium on, Aug. 1997, pp. 54-59. DOI: 10.1109/ISEMC. 1997. 667540 .

[8] T. Harrington, "GTEM fields FDTD modeling", in Electromagnetic Compatibility, 1997. IEEE 1997 International Symposium on, 1997, pp. 614-619. DOI: 10.1109/ISEMC.1997.667752. 